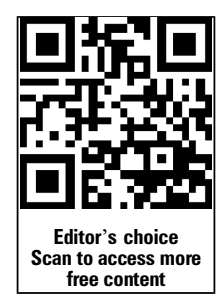

- Additional material is published online only. To view please visit the journal online (http://dx.doi.org/10.1136/ jmedgenet-2015-103027).

${ }^{1} \mathrm{CHU}$ Ste-Justine Research Center, Université de Montréal, Montréal, Québec, Canada ${ }^{2}$ Montreal Neurological Institute, McGill University, Montréal, Québec, Canada ${ }^{3}$ Istituto Giannina Gaslini, Genoa, Italy

\section{Correspondence to} Dr Zoha Kibar, Department of Neurosciences, University of Montréal, CHU Sainte Justine Research Center, 3175 CoteSte-Catherine, Room 5999C Montreal, Québec, Canada H3T 1C5. zoha.kibar@recherche-stejustine.qc.ca

Received 16 January 2015 Revised 26 February 2015 Accepted 4 March 2015 Published Online First 24 March 2015

\title{
Loss-of-function de novo mutations play an important role in severe human neural tube defects
}

\author{
Philippe Lemay, ${ }^{1}$ Marie-Claude Guyot, ${ }^{1}$ Élizabeth Tremblay, ${ }^{1}$ \\ Alexandre Dionne-Laporte, ${ }^{2}$ Dan Spiegelman, ${ }^{2}$ Édouard Henrion, ${ }^{2}$ Ousmane Diallo, ${ }^{2}$ \\ Patrizia De Marco, ${ }^{3}$ Elisa Merello, ${ }^{3}$ Christine Massicotte, ${ }^{1}$ Valérie Désilets, ${ }^{1}$ \\ Jacques L Michaud, ${ }^{1}$ Guy A Rouleau, ${ }^{2}$ Valeria Capra, ${ }^{3}$ Zoha Kibar ${ }^{1}$
}

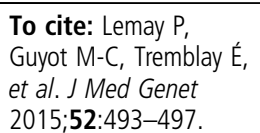

\section{ABSTRACT}

Background Neural tube defects (NTDs) are very common and severe birth defects that are caused by failure of neural tube closure and that have a complex aetiology. Anencephaly and spina bifida are severe NTDs that affect reproductive fitness and suggest a role for de novo mutations (DNMs) in their aetiology.

Methods We used whole-exome sequencing in 43 sporadic cases affected with myelomeningocele or anencephaly and their unaffected parents to identify DNMs in their exomes.

Results We identified 42 coding DNMs in 25 cases, of which 6 were loss of function (LoF) showing a higher rate of LoF DNM in our cohort compared with control cohorts. Notably, we identified two protein-truncating DNMs in two independent cases in SHROOM3, previously associated with NTDs only in animal models. We have demonstrated a significant enrichment of LoF DNMs in this gene in NTDs compared with the gene specific DNM rate and to the DNM rate estimated from control cohorts. We also identified one nonsense DNM in PAX3 and two potentially causative missense DNMs in GRHL3 and PTPRS.

Conclusions Our study demonstrates an important role of LoF DNMs in the development of NTDs and strongly implicates SHROOM3 in its aetiology.

Neural tube defects (NTDs) are a group of congenital malformations affecting 1-2 individuals per 1000 births. ${ }^{1}$ They are caused by an incomplete closure of the neural tube during embryogenesis. ${ }^{1}$ The most frequent forms of NTDs are anencephaly and myelomeningocele (MMC) (or spina bifida), which are caused by a closure defect in the brain and the spinal cord region, respectively. ${ }^{1}$ Children affected with anencephaly die early in development or soon after birth, while children affected with MMC survive but are affected by developmental physical defects with varying degrees of severity. Most cases of NTDs are sporadic and nonsyndromic. ${ }^{2}$ Periconceptional folic acid intake has been shown to reduce prevalence of NTDs by 50 $70 \%,{ }^{3}$ but a large amount of cases remain resistant to this preventive treatment urging the need for identification of other causative factors and development of novel preventive and counselling strategies.

NTDs have a strong genetic component with an estimated heritability of $60 \%,{ }^{1}$ but so far the genetics of the disease remains largely unknown. Previous linkage studies in NTDs have identified candidate regions on chromosomes 2, 7 and 10 but failed to identify any causative NTD gene. ${ }^{4}$ Few common variants in folic acid-related genes have also been shown to be associated with NTDs in certain populations, but these variants seem to contribute only to a small part of the aetiology of the disease. $^{5}$ Previous gene identification studies in NTDs have mainly adopted a candidate gene approach and focused on folate-related genes and on candidate genes from animal studies. ${ }^{1} \begin{array}{ccc}2 & 5\end{array}$ Animal models have demonstrated an important role of the planar cell polarity pathway in the aetiology of NTDs. ${ }^{1}$ Subsequent investigation of genes of this pathway, including VANGL1 and VANGL2, in human NTDs has implicated them as risk factors in a small fraction of patients. ${ }^{1}$ Generally, candidate gene studies in NTDs have faced limited success in identifying major causative genes predisposing to NTDs, suggesting the need for novel approaches. Several recent studies strongly suggest that de novo mutations (DNMs) represent a common cause of birth defects and neurodevelopmental diseases. ${ }^{6}$ DNM could provide a mechanism by which early-onset reproductively lethal diseases remain frequent in the population. Therefore, these variants are strong candidates for causing diseases that occur sporadically and that have a reduced reproductive fitness. ${ }^{6}$ Severe forms of NTDs, such as anencephaly and MMC, fall in this category and hence investigation of DNMs may therefore increase the chance of identifying loss of function (LoF) DNMs implicated in NTDs.

Forty-three families each composed of one affected child and two unaffected parents with no family history of NTDs were recruited through the Montreal Ste-Justine Hospital Spina Bifida Center, the 3D study of the Integrated Research Network in Perinatology of Quebec and Eastern Ontario and the Istituto Giannina Gaslini in Genoa, Italy. Detailed information including folate status, tissue of origin and type of NTDs of this cohort is summarised in online supplementary table S1. Briefly, all 43 cases were affected with NTDs including $35 \mathrm{MMC}$ and 8 anencephaly cases. A total of 21 cases were fetuses and 55.6\% took folate periconceptionally. Tissues from fetuses were all obtained following induced abortions. The average maternal and paternal ages were 30.0 \pm 4.8 years and $30.7 \pm 5.9$ years, respectively. 
Exome data from 43 NTD trios were aligned to the GRCh37 genome reference and called variants were filtered for minimal quality (genotype quality (GQ) $>20$, total variant reads $>3$, total reads $>7)$ and for absence in parents and public databases (1000 genome at http://www.1000genomes.org/home and Exome Variant Server at http://evs.gs.washington.edu/EVS/) to identify DNMs. Details of the alignment strategy and singlenucleotide variant (SNV) annotation are included in online supplementary data. Seventy variants were identified and validated by Sanger sequencing to minimise false negative results. This approach resulted in the identification of 42 coding mutations in 25 trios including 3 nonsense, 3 frameshift, 30 missense and 6 synonymous (table 1 ). The average DNM rate per base pair was $1.62 \times 10^{-8}$, which was consistent with published ratios. ${ }^{78}$

We identified six LoF DNMs defined as nonsense, frameshift and splicing variants, in six NTD trios resulting in a per base DNMs rate of $0.23 \times 10^{-8}$. This rate was higher than two previously published per base LoF DNMs rates, $0.05 \times 10^{-8}$ (ref. 8) and $0.17 \times 10^{-8}$ (ref. 9) but only reached significance $(\mathrm{p}=0.002)$ when compared to the lower rate, suggesting that larger cohorts

Table 1 De novo mutations identified in NTD-affected trios and confirmed by Sanger sequencing.

\begin{tabular}{|c|c|c|c|c|c|c|c|}
\hline Family & NTD type & Chr & Position & Genes & cDNA change & Amino acid change & Polyphen HDIV* \\
\hline \multicolumn{8}{|c|}{ Loss-of-function de novo mutations (nonsense, frameshift, splicing) } \\
\hline $\operatorname{Pr} 394$ & MMC & 4 & 77662169 & SHROOM3 & c.2843_2844insG & p.L948fs & NA \\
\hline PrZRV & Anen & 4 & 77660502 & SHROOM3 & C. $1176 C>G$ & p.Y392X & NA \\
\hline $\operatorname{Pr} 134$ & MMC & 2 & 223161800 & PAX3 & c. $218 C>A$ & p.S73X & NA \\
\hline Pr201 & MMC & 15 & 44116692 & MFAP1 & c.69del7 & p.K23fs & NA \\
\hline $\operatorname{Pr} 389$ & MMC & $X$ & 41202544 & $D D \times 3 X$ & c.620dupA & p.Q207fs & NA \\
\hline PrYPT & MMC & 7 & 73279630 & WBSCR28 & C. $380 \mathrm{G}>\mathrm{A}$ & p.W127X & NA \\
\hline \multicolumn{8}{|c|}{ Missense and synonymous de novo mutations } \\
\hline $\operatorname{Pr} 548$ & MMC & 1 & 24668728 & GRHL3 & c. $1171 \mathrm{C}>\mathrm{T}$ & p.R391C & 1 \\
\hline $\operatorname{Pr} 548$ & MMC & 19 & 39798985 & LRFN1 & c. $1604 C>T$ & p.T535I & 1 \\
\hline $\operatorname{Pr} 548$ & MMC & 9 & 124751686 & TTLL11 & c. $1327 A>G$ & p.N443D & 0.968 \\
\hline $\operatorname{Pr} 125$ & MMC & 19 & 5214591 & PTPRSt & c. $4475 G>A$ & p.R1049Q & 0.998 \\
\hline $\operatorname{Pr} 125$ & MMC & 2 & 27435209 & ATRAID & c. $138 \mathrm{G}>\mathrm{A}$ & p.A46A & NA \\
\hline $\operatorname{Pr} 122$ & MMC & 12 & 123341629 & $H I P 1 R$ & c. $1682 \mathrm{G}>\mathrm{T}$ & p.G561V & 0.019 \\
\hline $\operatorname{Pr} 122$ & MMC & 17 & 71232301 & C17orf80 & c. $1441 C>T$ & p.R481W & 0.099 \\
\hline $\operatorname{Pr} 122$ & MMC & $X$ & 53592096 & HUWE1 & c. $6812 \mathrm{G}>\mathrm{A}$ & p.S2271N & 0 \\
\hline Pr134 & MMC & 14 & 105179874 & INF2† & c. $2971 C>T$ & p.R991W & 1 \\
\hline Pr191 & MMC & 8 & 10464772 & RP1L1† & c. $6836 C>T$ & p.P2279L & 0.053 \\
\hline $\operatorname{Pr} 191$ & MMC & 20 & 62371335 & $S L C 2 A 4 R G$ & c. $70 \mathrm{C}>\mathrm{T}$ & p.R24C & 0.426 \\
\hline Pr20 & MMC & 3 & 38317786 & $S L C 22 A 13$ & c. $1246 \mathrm{G}>\mathrm{A}$ & p.V416M & 0.948 \\
\hline Pr20 & MMC & 16 & 31383022 & ITGAX & c. 2077 C >G & p.Q693E & 0.001 \\
\hline Pr201 & MMC & 2 & 47703654 & MSH2† & c. $1956 \mathrm{~A}>\mathrm{G}$ & p.Q652Q & NA \\
\hline Pr202 & MMC & 6 & 30122164 & TRIM10 & c. $1028 \mathrm{~A}>\mathrm{T}$ & p.D343V & 1 \\
\hline Pr202 & MMC & 7 & 23775208 & STK31 & c. $535 \mathrm{~A}>\mathrm{C}$ & p.I179L & 0.039 \\
\hline Pr25 & MMC & 9 & 134183554 & PPAPDC3† & c. $696 C>T$ & p.12321 & $\mathrm{NA}$ \\
\hline Pr263 & MMC & 6 & 1390351 & FOXF2 & c. $169 \mathrm{G}>\mathrm{A}$ & p.A57T & 0.01 \\
\hline Pr28 & MMC & 8 & 124333387 & ATAD2 & c. $4160 \mathrm{G}>\mathrm{A}$ & p.S1387N & 0.001 \\
\hline Pr282 & MMC & 19 & 808439 & PTBP1 & c. $1233 C>A$ & p.N411K & 0.013 \\
\hline Pr389 & MMC & 7 & 100285176 & GIGYF1 & c. $325 C>T$ & p.P109s & 0.728 \\
\hline Pr402 & MMC & 17 & 19319353 & RNF112 & c. $1761 C>T$ & p.A587A & NA \\
\hline Pr402 & MMC & 3 & 49775724 & IP6K1 & c. $355 C>T$ & p.R119C & 1 \\
\hline Pr402 & MMC & 4 & 48424093 & SLAIN2 & c. $1745 G>C$ & p.X582S & NA \\
\hline $\operatorname{Pr} 530$ & MMC & 3 & 57616163 & DENND6A & c. $1605 \mathrm{~A}>\mathrm{C}$ & p.E535D & 0.997 \\
\hline Pr551 & MMC & 9 & 130279261 & FAM129B & c. $848 \mathrm{C}>\mathrm{T}$ & p.A283V & 0.004 \\
\hline $\operatorname{Pr} 553$ & MMC & 19 & 39329153 & HNRNPL & c. $1441 C>T$ & p.R481W & 1 \\
\hline Pr554 & Anen & 3 & 142741447 & U2SURP & c. $961 \mathrm{G}>\mathrm{A}$ & p.G321s & 1 \\
\hline Pr554 & Anen & 5 & 93966388 & ANKRD32 & c. $371 \mathrm{~T}>\mathrm{C}$ & p.F124S & 0.999 \\
\hline Pr67 & MMC & 2 & 44566318 & PREPL & c. $937 C>G$ & p.L313V & 1 \\
\hline Pr67 & MMC & 5 & 176314262 & HK3 & c. $1677 \mathrm{G}>\mathrm{A}$ & p.V559V & NA \\
\hline Pr67 & MMC & 9 & 33264606 & $B A G 1$ & c. $67 \mathrm{G}>\mathrm{A}$ & p.A23T & 0.897 \\
\hline PrKKS & MMC & 15 & 43621819 & LCMT2† & c. $869 \mathrm{~T}>\mathrm{C}$ & p.1290T & 0.201 \\
\hline PrKKS & MMC & 7 & 11076097 & PHF14 & c. $1655 G>C$ & p.R552P & 0.998 \\
\hline PrTVB & MMC & 22 & 41558745 & EP300† & c. $3690 A>G$ & p.Q1230Q & NA \\
\hline PrVWA & MMC & 9 & 120475791 & TLR4 & c. $1385 C>T$ & p.A462V & 0.006 \\
\hline
\end{tabular}

*Probably damaging (polyphen HDIV $\geq 0.957$ ), possibly damaging $(0.453 \leq$ polyphen HDIV $\leq 0.956$ ); benign (polyphen HDIV $\leq 0.452$ ).

tMutation previously reported in the ExAC database (http:/lexac.broadinstitute.org/). Reported mutations frequencies are PTPRS (frequency: 0.000008368 ); INF2 (frequency:

0.000009451); RP1L1 (frequency: 0.0001411); MSH2 (frequency: 0.0001730), PPAPDC3 (frequency: 00003366); LCMT2 (frequency: 0.000008281); EP300 (frequency: 0.0000082).

Anen, anencephaly; MMC, myelomeningocele; NA, non-applicable; NTD, neural tube defect. 
of MMC and anencephaly are needed to confirm these initial findings. This increased rate suggests that LoF DNMs are an important part of the pathogenicity of NTDs. Details of all statistical analysis can be found in online supplementary data.

Notably, we identified two LoF DNMs in SHROOM3 (NM 020859.3) in two unrelated families: one nonsense variant c.1176C $>$ G (p.Y392X) and one frameshift variant c.2843_2844insG leading to a premature stop codon (p.L948fs) (figure 1B). The c.1176C $>\mathrm{G}$ variant was detected in the PrZRV proband affected with anencephaly with cranial fossa agenesis and facial dysmorphism (table 1). The mother took folic acid periconceptionally (see online supplementary table S1). The c.2843_2844insG variant was detected in the Pr394 proband affected with a thoracic MMC and Chiari type IV malformation (table 1). The mother did not take folic acid periconceptionally (see online supplementary table S1). None of these mutations were reported in the ExAC database (http://exac.broadinstitute. org/). SHROOM3 is an actin-binding protein that is known to be a key regulator of apical constriction, a process by which cells convert their shape from cuboidal to wedge-like due to a decrease in their apical area. ${ }^{9}$ This function is essential for hingepoint formation and bending of the neural tube during its formation and closure in both vertebrate embryos. ${ }^{9}$ Recessive mutations in SHROOM3 were previously associated to heterotaxy, which represents a multiple congenital anomaly syndrome resulting from abnormalities of the proper specification of left-right asymmetry during embryonic development. ${ }^{10}$ The LoF DNM in this gene described in our study was detected in two patients with NTD who showed no sign of heterotaxy, and hence these NTD mutations might act in a haploinsufficient or a dominant negative manner. Shroom 3 exists in two isoforms that were demonstrated to have similar functions and expression patterns in the mouse and frog models. ${ }^{11}{ }^{12}$ In the mouse gene trap mutant, both isoforms are knocked out, leading to exencephaly and spina bifida in homozygous embryos at a penetrance of $100 \%$ and $23 \%$, respectively. ${ }^{11}$ In our cohort, both truncating DNMs detected in SHROOM3 seem to affect both isoforms since they map at positions 1176 and $2843 \mathrm{bp}$ respectively $5^{\prime}$ or inside the ASD1 domain (figure 1A). These two DNMs clearly remove important functional domains and might confer nonsense-mediated RNA decay and hence they are most likely LoF mutations. Furthermore, previous studies have demonstrated the potential of a truncated version of Shroom 3 to act in a dominant negative manner in Xenopus. ${ }^{12}$ This supports the potential pathogenicity of these heterozygous mutations that could result in limited apical constriction causing the NTD.

The phenotypic variation between the two probands (MMC and anencephaly) who carry LoF DNM in SHROOM3 could be
A

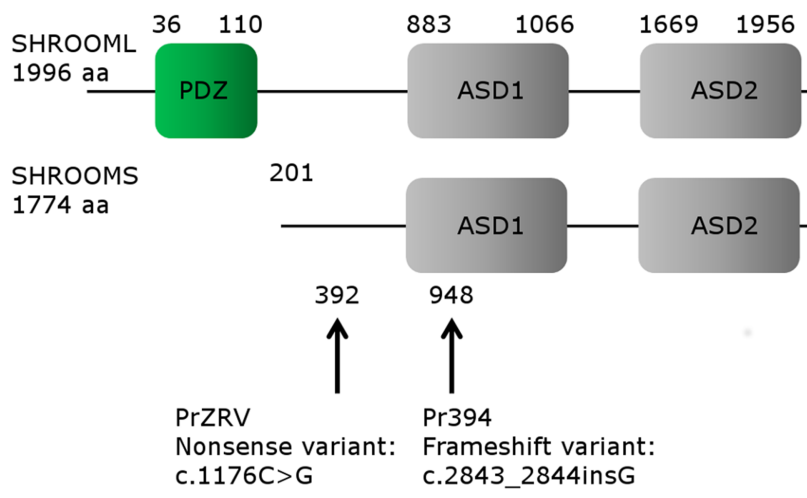

B
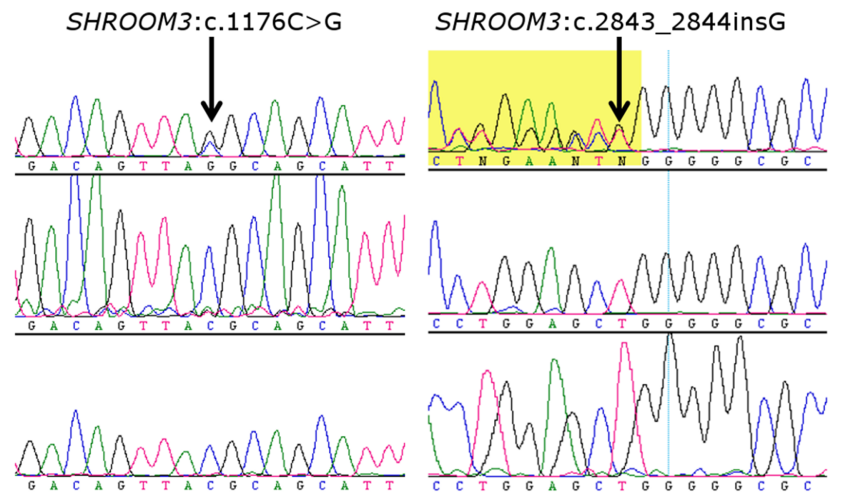

C

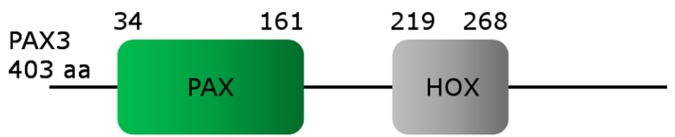

79

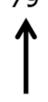

Pr134

Nonsense variant:

c. $218 \mathrm{C}>\mathrm{A}$

Figure 1 De novo mutations (DNMs) identified in SHROOM3 and in PAX3 in trios affected with neural tube defects (NTDs). (A) Protein schematic representation of the long and predicted short isoforms of SHROOM3 indicating the position of the two DNMs identified in NTD probands. (B) Chromatograms of the three individuals of family PrZRV and Pr394 (from top to bottom: proband, mother, father) surrounding the SHROOM3 c.1176C>G nonsense variant and the c.2843_2844insG frameshift variant. (C) Protein schematic representation of the long and predicted short isoforms of PAX3 indicating the position of the DNM identified in NTD probands. (D) Chromatograms of the three individuals of family Pr134 (from top to bottom: proband, mother, father) surrounding the PAX3 c.218C >A nonsense variant. HOX, paired-type homeodomain; PAX, paired box domain. 
caused by many factors including genetic modifiers and environmental factors. The two variants might also have different pathogenic effects on the protein with different outcomes in the implicated developmental pathway. These factors could act alone or in combination to affect the clinical expressivity of the NTD phenotype.

Considering the estimated LoF DNM rates between $0.05 \times 10^{-8}$ and $0.17 \times 10^{-8} \mathrm{SNV}$ per base ${ }^{8}$ and the length of SHROOM3 long isoform (NM_020859.3: $5991 \mathrm{bp}$ ), we expect to find respectively between 0.00026 and 0.00088 LoF DNM in this gene in our cohort. However, we detected two DNMs in our cohort of 43 trios, resulting in a frequency of DNM per bp of $3.88 \times 10^{-6}$. This represents a significant enrichment of LoF DNMs in SHROOM3 under the two rates with respective Bonferroni corrected $\mathrm{p}$ values of $6.77 \times 10^{-4}$ and 0.008 using a two-tailed binomial exact test. A recently published gene-specific per trio mutation rate for LoF DNMs was also used to test the significant enrichment of LoF DNMs in SHROOM $3 .{ }^{13}$ Under the $S H R O O M 3$ gene-specific rate of $1.34 \times 10^{-5}$, we expected to find 0.00058 LoF DNMs in out cohort, which was significantly lower than our observed rate ( $p$ Bonferroni=0.003). Details of all statistical analyses can be found in online supplementary data.

Also importantly we detected one de novo stopgain in the NTD-associated gene PAX3, c.218C>A (p.S73X), in the Pr134 case affected with MMC with type II Arnold-Chiari malformation, hydrocephalus and Waardenburg syndrome (WS) (figure 1D). The mother did not take folic acid or multivitamins periconceptionally (see online supplementary table S1). This mutation was not reported in the ExAC database. PAX3 is a paired box transcription factor that is a key developmental regulator of the neural crest and its derivatives and that plays an important role during neurogenesis and myogenesis. It contains two DNA binding domains, the paired box domain (PAX) and a paired-type homeodomain (HOX) that interact cooperatively for DNA binding. ${ }^{14}$ The putative truncated protein caused by the DNM p.S73X lacks all functional domains including both PAX and HOX (figure 1C) and might confer nonsense-mediated RNA decay.

In the Splotch mouse model, homozygous LoF mutations of Pax 3 cause spina bifida and other neural crest abnormalities. In humans, the relative contribution of PAX3 to the overall burden of NTDs remains unclear. ${ }^{14}$ Heterozygous mutations in PAX3 are known to cause WS, an autosomal-dominant condition that affects neural crest-derived structures and that is occasionally associated with NTDs. Few individuals with both WS and NTDs were demonstrated to carry heterozygous PAX3 mutations or deletions. ${ }^{14}$ Our report of a new protein truncating SNV in PAX3 in one patient with NTD provides additional evidence for a pathogenic role of this gene in spina bifida.

While SHROOM3 and PAX3 are by far the strongest candidate genes identified in this study, other interesting DNMs have been identified in our cohort (table 1). We detected three LoF DNMs, p.W127X in WBSCR28, p.K23fs in MFAP1 and p. Q207fs in DDX3X, with no previous association to NTDs and that were not reported in the ExAC database (table 1, see online supplementary figure S1). The WBSCR28 DNM was detected in the PrYPT proband affected with lumbosacral MMC and type II Arnold-Chiari malformation. This gene encodes a putative transmembrane protein of unknown function that maps to the region deleted in the Williams-Beuren syndrome (table 1). This syndrome is characterised by a range of phenotypes including mental retardation, dysmorphic facies, heart abnormalities, short stature and infantile hypocalcaemia. WBSCR28 is not the main candidate gene in this disease. ${ }^{15}$ The MFAP1 DNM was found in the Pr201 proband affected with lumbosacral MMC. This gene encodes the microfibrillar-associated protein 1 that represents an uncharacterised protein found in some human spliceosomal fractions. ${ }^{16}$ The DDX3X DNM was found in the Pr389 proband affected with lumbosacral MMC and hydrocephalus. This gene belongs to the DEAD-box proteins, a large family of ATP-dependent RNA helicases that participate in all aspects of RNA metabolism. ${ }^{17}$ While none of these three genes represent strong candidates for NTDs based on published data, the presence of LoF DNMs in these genes still suggests them as potentially interesting NTD candidates. Additional genetic studies in larger cohorts and functional studies are needed to validate their role in NTDs.

Two other interesting missense DNM identified in two NTD trios implicated genes whose orthologues cause NTD in mice: c.1171C > T (p.R391C) in GRHL3 (GRAINYHEAD-LIKE 3) and c.4475G>A (p.R1492Q) in PTPRS (PROTEIN TYROSINE PHOSPHATASE, RECEPTOR TYPE, $S$ ) (see online supplementary figure S2). The GRHL3 mutation was identified in the Pr548 proband affected with MMC with type II ArnoldChiari malformation and hydromyelia. This mutation was not previously reported in the ExAC database. Grhl3 is a transcription factor that plays an important role in epidermal integrity and wound healing. Importantly, null alleles at this gene caused mainly severe spina bifida and occasionally exencephaly. Grhl3 was tightly linked to Curly tail that represents one of the most well-established mouse models for NTDs. ${ }^{18}$ The p.R391C maps to the DNA binding domain of the GHRL3 protein. The modified amino acid is highly conserved (see online supplementary figure S2), and the mutation is defined as probably damaging by PolyPhen-2 HDIV (table 1). Surprisingly, the same DNM p. R391C was detected in Van der Woude syndrome, the most common syndromic form of cleft lip and palate. ${ }^{19}$ While this could point towards a chance finding, we hypothesise that additional genetic and/or environmental factors may modify the phenotypic expression of the same GRHL3 mutation in different individuals. The p.R1492Q in PTPRS was found in the Pr125 proband affected with lumbosacral MMC with type II Arnold-Chiari malformation. The mutation was previously reported on one allele in the ExAC database (http://exac. broadinstitute.org/), resulting in a frequency of 0.000008368 . This gene is a phosphatase involved in regulating cell proliferation, cell adhesion and nervous system maturation and causes exencephaly in mice. ${ }^{20}$ PTPRS fibronectin and Ig-like domains are extracellular and mainly involved in cell interaction, while the catalytic phosphatase domain is intracellular and mainly involved in signal transmission. The p.R1049Q variant was identified in a proband affected with MMC (table 1) and resides in a conserved region that forms part of the phosphatase domain (see online supplementary figure S2). It modifies a conserved arginine to a glutamine, a non-conservative substitution that removes the positive charge of the amino acid side chain, changes its size and is predicted to be probably damaging by PolyPhen-2 (table 1).

Molecular genetic studies of bigger cohorts and careful phenotyping are still needed to better understand the mechanism of action of potential DNMs detected in this study and to assess their role in the pathogenicity in NTDs. While these DNMs are most likely highly penetrant, it is possible that they act in concert with other events to cause the disease. This is consistent with the two-hit model that was initially proposed as a cancer mechanism and later suggested as a potential mechanism in complex diseases. ${ }^{21}$ This model was more recently explored in 
explaining variable expressivity in severe developmental genomic disorders. ${ }^{21}$ In this model, a secondary insult is necessary to result in a different or more severe clinical manifestation of a complex disease. This insult occurs during development and could be genetic or epigenetic (germ line or somatic) or environmental. The two 'hits' could act independently or additively to each other, resulting in a phenotype that differs from either hit alone. Alternatively, the two hits could involve gene(s) from the same or similar biochemical pathway and hence could interact in an epistatic manner.

The novel approach of whole-exome sequencing has long been due in the genetic investigation of NTDs. This study uses this powerful approach in this complex trait and has successfully identified potential candidate DNMs in novel genes in the development of human NTDs. We have demonstrated the presence of LoF variants in five genes, have reported more LoF DNMs in our cohort than expected and have identified two of those mutations in orthologues of mouse NTD genes, suggesting the involvement of those variants in the aetiology of human NTDs. We have also demonstrated the presence of two independent protein truncating variants in SHROOM3 in 43 trios and presented a statistically significant enrichment of LoF DNMs in this gene compared with control and gene-specific DNMs rates. Our data strongly suggest that highly penetrant pathogenic variants in this gene may account for a significant part of the genetic aetiology of severe forms of NTD. Further studies of this gene in a bigger cohort of sporadic cases may help better assess the significance of these findings.

Acknowledgements We would like to thank all participants in this study.

Contributors PL, JLM, GAR, VC and ZK designed the study. PL, M-CG and ET validated the mutations. PL, AD-L, DS, EH and OD analysed the genetic data. PL ran the statistical analysis. PDM, EM, CM, VD, VC and ZK recruited patients and provided clinical information. PL and ZK wrote the manuscript.

Funding CHU Ste-Justine fundation; Fonds de Recherche du Québec—Santé; Canadian Institutes of Health Research. This project was conducted as part of the research programme of the Integrated Research Network in Perinatology of Quebec and Eastern Ontario (IRNPQEO). This work was supported by the Canadian Institutes of Health Research (CRI 88413 and MOP 130411).

Competing interests ZK has a salary award from the "Fonds de Recherche du Québec-Santé". JLM is a National Scientist of the Fonds de Recherche du Québec-Santé. PL is supported by "Fondation du CHU Ste-Justine" and "Fonds de Recherche du Québec-Santé". Authors have no competing interest.

Patient consent Obtained.

Ethics approval CHU Sainte Justine Hospital (Protocols' numbers: 2598 and 2899) and Istituto Giannina Gaslini, Genoa, Italy (protocol number: 213/2013).

Provenance and peer review Not commissioned; externally peer reviewed.

\section{REFERENCES}

1 Bassuk AG, Kibar Z. Genetic basis of neural tube defects. Semin Pediatr Neurol 2009;16:101-10.

2 Copp AJ, Stanier P, Greene ND. Neural tube defects: recent advances, unsolved questions, and controversies. Lancet Neurol 2013;12:799-810.
3 [No authors listed]. Prevention of neural tube defects: results of the Medical Research Council Vitamin Study. MRC Vitamin Study Research Group. Lancet 1991;338:131-7.

4 Stamm DS, Siegel DG, Mehltretter L, Connelly JJ, Trott A, Ellis N, Zismann V, Stephan DA, George TM, Vekemans M, Ashley-Koch A, Gilbert JR, Gregory SG, Speer MC. Refinement of $2 q$ and $7 p$ loci in a large multiplex NTD family. Birt Defects Res A Clin Mol Teratol 2008;82:441-52.

5 Zhang T, Lou J, Zhong R, Wu J, Zou L, Sun Y, Lu X, Liu L, Miao X, Xiong G. Genetic variants in the folate pathway and the risk of neural tube defects: a meta-analysis of the published literature. PloS ONE 2013;8:e59570.

6 Veltman JA, Brunner HG. De novo mutations in human genetic disease. Nat Rev Genet 2012:13:565-75.

7 Sanders SJ, Murtha MT, Gupta AR, Murdoch JD, Raubeson MJ, Willsey AJ, Ercan-Sencicek AG, DiLullo NM, Parikshak NN, Stein JL, Walker MF, Ober GT, Teran NA, Song Y, El-Fishawy P, Murtha RC, Choi M, Overton JD, Bjornson RD, Carriero NJ, Meyer KA, Bilguvar K, Mane SM, Sestan N, Lifton RP, Günel M, Roeder K, Geschwind DH, Devlin B, State MW. De novo mutations revealed by whole-exome sequencing are strongly associated with autism. Nature 2012;485:237-41.

8 Iossifov I, O'Roak BJ, Sanders SJ, Ronemus M, Krumm N, Levy D, Stessman HA Witherspoon KT, Vives L, Patterson KE, Smith JD, Paeper B, Nickerson DA, Dea J, Dong S, Gonzalez LE, Mandell JD, Mane SM, Murtha MT, Sullivan CA, Walker MF, Waqar Z, Wei L, Willsey AJ, Yamrom B, Lee Y, Grabowska E, Dalkic E, Wang Z, Marks S, Andrews P, Leotta A, Kendall J, Hakker I, Rosenbaum J, Ma B, Rodgers L, Troge J, Narzisi G, Yoon S, Schatz MC, Ye K, McCombie WR, Shendure J, Eichler $E E$, State MW, Wigler M. The contribution of de novo coding mutations to autism spectrum disorder. Nature 2014;515:216-21.

9 Sawyer JM, Harrell JR, Shemer G, Sullivan-Brown J, Roh-Johnson M, Goldstein B. Apical constriction: a cell shape change that can drive morphogenesis. Dev Biol 2010;341:5-19.

10 Tariq M, Belmont JW, Lalani S, Smolarek T, Ware SM. SHROOM3 is a novel candidate for heterotaxy identified by whole exome sequencing. Genome Biol 2011;12:R91.

11 Hildebrand JD, Soriano P. Shroom, a PDZ domain-containing actin-binding protein, is required for neural tube morphogenesis in mice. Cell 1999;99:485-97.

12 Haigo SL, Hildebrand JD, Harland RM, Wallingford JB. Shroom induces apical constriction and is required for hingepoint formation during neural tube closure. Curr Biol CB 2003;13:2125-37

13 Samocha KE, Robinson EB, Sanders SJ, Stevens C, Sabo A, McGrath LM, Kosmicki JA, Rehnström K, Mallick S, Kirby A, Wall DP, MacArthur DG, Gabriel SB, DePristo M, Purcell SM, Palotie A, Boerwinkle E, Buxbaum JD, Cook EH, Gibbs RA, Schellenberg GD, Sutcliffe JS, Devlin B, Roeder K, Neale BM, Daly MJ. A framework for the interpretation of de novo mutation in human disease. Nat Genet 2014;46:944-50.

14 Greene NDE, Massa V, Copp AJ. Understanding the causes and prevention of neural tube defects: Insights from the splotch mouse model. Birt Defects Res A Clin Mol Teratol 2009;85:322-30.

15 Hockenhull EL, Carette MJ, Metcalfe K, Donnai D, Read AP, Tassabehji M. A complete physical contig and partial transcript map of the Williams syndrome critical region. Genomics 1999;58:138-45.

16 Hegele A, Kamburov A, Grossmann A, Sourlis C, Wowro S, Weimann M, Will CL, Pena V, Lührmann R, Stelzl U. Dynamic protein-protein interaction wiring of the human spliceosome. Mol Cell 2012;45:567-80.

17 Soto-Rifo R, Ohlmann T. The role of the DEAD-box RNA helicase DDX3 in mRNA metabolism. Wiley Interdiscip Rev RNA 2013;4:369-85.

18 Gustavsson P, Copp AJ, Greene NDE. Grainyhead genes and mammalian neural tube closure. Birt Defects Res A Clin Mol Teratol 2008;82:728-35

19 Peyrard-Janvid M, Leslie EJ, Kousa YA, Smith TL, Dunnwald M, Magnusson M, Lentz BA, Unneberg P, Fransson I, Koillinen HK, Rautio J, Pegelow M, Karsten A, Basel-Vanagaite L, Gordon W, Andersen B, Svensson T, Murray JC, Cornell RA, Kere J, Schutte BC. Dominant mutations in GRHL3 cause Van der Woude Syndrome and disrupt oral periderm development. Am J Hum Genet 2014;94:23-32.

20 Uetani N, Bertozzi K, Chagnon MJ, Hendriks W, Tremblay ML, Bouchard M. Maturation of ureter-bladder connection in mice is controlled by LAR family receptor protein tyrosine phosphatases. J Clin Invest 2009;119:924-35.

21 Kumar RA. Two-hit wonder: a novel genetic model to explain variable expressivity in severe pediatric phenotypes. Clin Genet 2010;78:517-9. 